\title{
Monostotic Fibrous Dysplasia of the Left Parietal Bone - Case Report-
}

\author{
Shin-ichi Otsuka, Shoji NaKatsu, Shigeo Matsumoto, Shin-ichi Sato, \\ Takahiko MOTOZAKI, Sadahiko BAN, Toyoshiro YAMAMOTO, \\ Hirofumi SHIRANE*, Shigeo SAIWAI** and Satoshi NAKAO***
}

\author{
Departments of Neurosurgery, ${ }^{*}$ Clinical Pathology, and ${ }^{* *}$ Radiology, \\ Kobe City General Hospital, Kobe; ${ }^{* * *}$ Department of Neurosurgery, \\ Kishiwada City Hospital, Kishiwada, Osaka
}

\begin{abstract}
A 14-year-old female presented with a hard, painless mass, $5 \times 5 \mathrm{~cm}$, in the left parietal region. Skull $\mathrm{x}$-rays showed a radiolucent skull tumor with a sclerotic margin in the parietal region. Computed tomography revealed an intradiploic multilocular mass separated by bony trabeculae. The outer table had thinned and protruded outward. The inner table was also thin and protruded inward slightly. External carotid angiography revealed a faint tumor stain and feeding from the middle meningeal artery. Bone scintigraphy revealed abnormal uptake in the lesion. Total removal of the skull tumor and cranioplasty were performed. The histological diagnosis was fibrous dysplasia. Fibrous dysplasia within the cranial vault is often expressed as painless bulging without neurological symptoms. Surgery is recommended when neurological symptoms and/or cosmetic problems are present. Histological confirmation of the diagnosis is also important.
\end{abstract}

Key words: monostotic fibrous dysplasia, cranial vault, external carotid angiography, tumor stain

\section{Introduction}

Fibrous dysplasia involves abnormal differentiation of the bone-forming mesenchyme. The etiology is unknown, but it is often encountered in adolescents. The craniofacial skeleton is one of its predilective sites, and it often occurs in the skull base and facial bones. In cases of polyostotic fibrous dysplasia, the lesions also occur in the cranial vault, whereas monostotic fibrous dysplasia rarely develops in the cranial vault. We treated a patient with monostotic fibrous dysplasia of the cranial vault whose chief complaint was a left parietal mass. We describe this case and discuss the relevant literature.

\section{Case Report}

A 14-year-old female noticed a hard, painless mass in the left parietal region in April of 1986. It gradually enlarged, and she was hospitalized on January

Received May 2, 1988; Accepted July 11, 1988

19, 1987. On admission, a physical examination revealed a hard, nontender mass of $5 \times 5 \mathrm{~cm}$ in the left parietal region.

Skull $\mathrm{x}$-rays demonstrated a radiolucent skull tumor with a sclerotic margin in the left parietal bone (Fig. 1). Computed tomography (CT) revealed

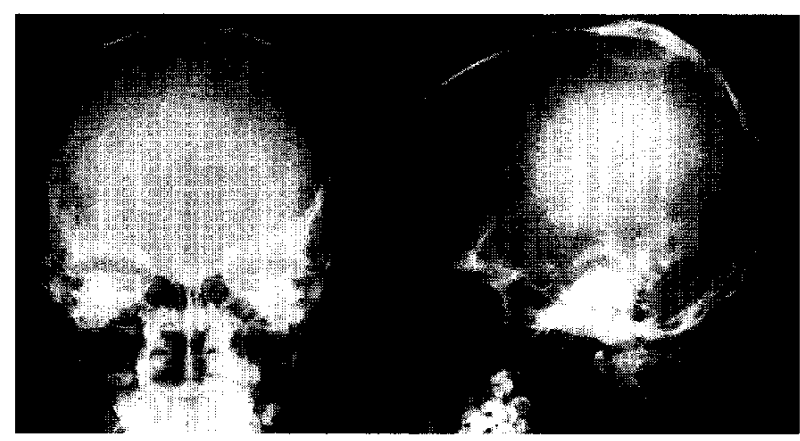

Fig. 1 Skull x-rays demonstrating a round, radiolucent tumor in the left parietal bone. 
an intradiploic, multilocular mass separated by bony trabeculae. The outer table was thin and protruded outward. The inner table was also thin and protruded inward slightly (Fig. 2). Left external carotid angiography revealed a faint tumor stain in the left parietal region. The tumor was fed by the middle meningeal artery (Fig. 3). Bone scintigraphy revealed abnormal uptake within the mass but no abnormalities in any other bones (Fig. 4).

These neuroradiological findings suggested fibrous dysplasia of the left parietal bone. She underwent surgery for removal of the cosmetic deformity and histological confirmation of the diagnosis. Retraction of the scalp in the left parietal region revealed a pale, pinkish, bony mass $5 \times 5 \mathrm{~cm}$ in area.

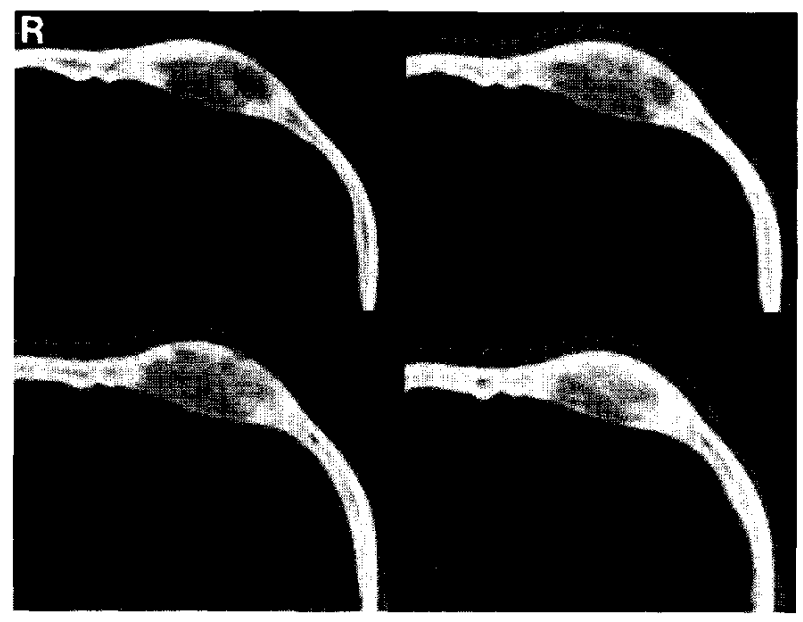

Fig. 2 CT scans revealing an intradiploic, multilocular mass separated by some bony trabeculae. The outer table is thin and protrudes outward. The inner table is also thin and protrudes inward slightly.

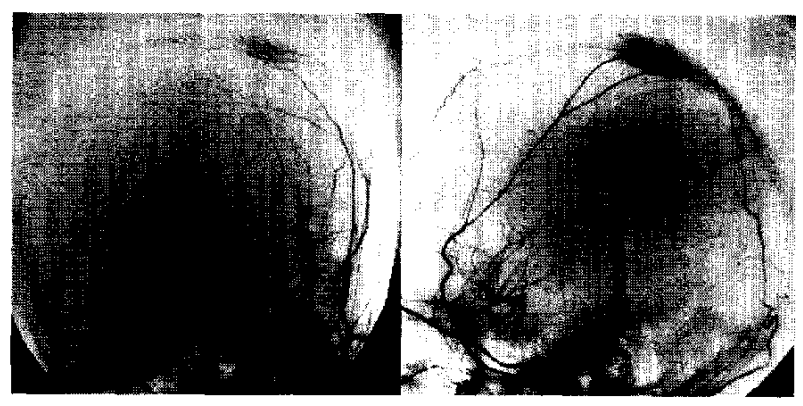

Fig. 3 Left external carotid angiograms, showing a faint tumor stain in the left parietal region, with feeding from the middle meningeal artery.
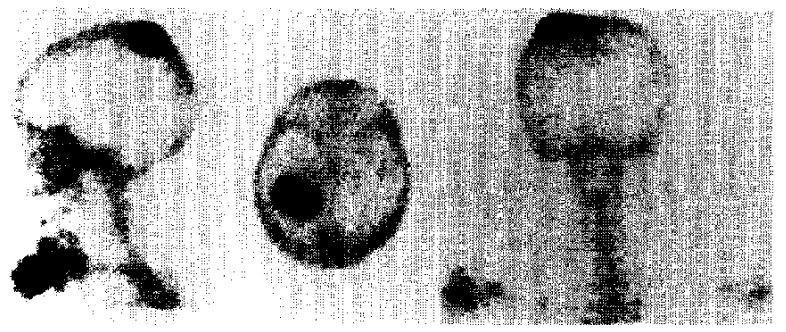

Fig. 4 Bone scintigrams demonstrating abnormal uptake by the left parietal lesion.
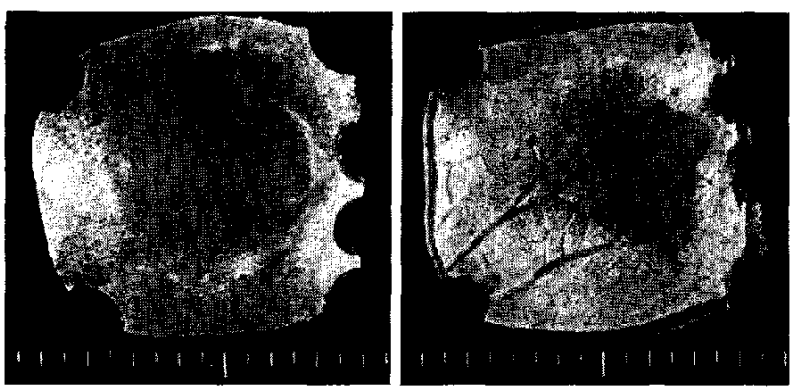

Fig. 5 In the resected bone, pathological changes were mainly confined to the outer table. Its color had changed to pale pink and it protruded outward markedly (left). The inner table was somewhat affected; it was also pale pink and protruded inward slightly (right).

The bony mass and the normal bone surrounding it were resected. Pathological changes were found mainly in the outer table, which was pale pink and protruded outward markedly. There was also some pathological change in the inner table, which was pale pink and protruded inward slightly (Fig. 5). The bone defect was repaired with a titanium plate.

Histological examination of the resected tissue revealed fibrous dysplasia consisting of a proliferation of fibrous connective tissue associated with bony trabeculae of various shapes and sizes (Fig. 6).

\section{Discussion}

Fibrous dysplasia is a skeletal disorder of unknown etiology, characterized by replacement of normal bone substance by abnormal fibro-osseous connective tissue. The onset is usually within the first three decades of life, and more females than males are affected. ${ }^{9)}$ Fibrous dysplasia is classified as monostotic, in which a single bone is affected, and polyostotic, which involves many bones. In many cases of the polyostotic type, the bones are affected 


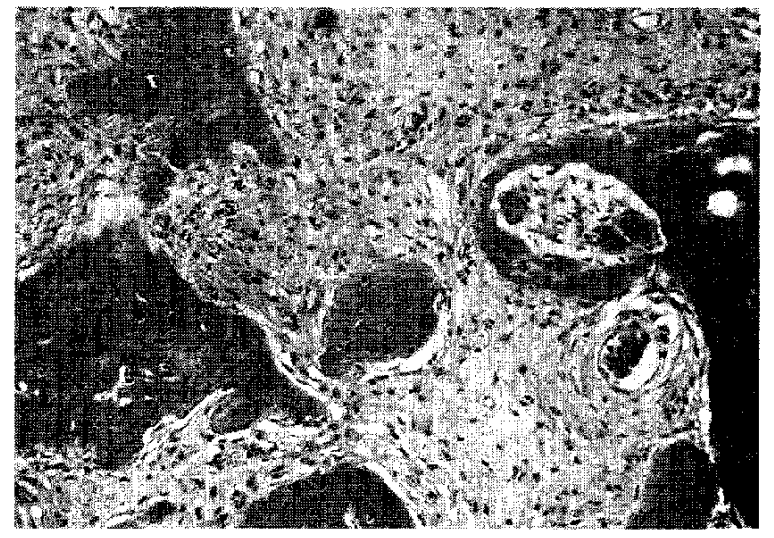

Fig. 6 Photomicrograph of the surgical specimen, showing fibrous dysplasia consisting of proliferation of fibrous connective tissue and bony trabeculac of various shapes and sizes. HE stain, $\times 100$.

unilaterally. Skull involvement is commoner in polyostotic fibrous dysplasia, ${ }^{11}$ and the frontal bone is most frequently affected, followed by the sphenoid bone. Together, these two bones constitute the majority of cases of cranial fibrous dysplasia. ${ }^{13)}$

Monostotic fibrous dysplasia of the parietal bone is rare. The most frequent clinical symptom is a local, hard protrusion, which is usually painless and rarely causes neurological abnormalities. ${ }^{1)}$ However, there have been reports of cranial monostotic fibrous dysplasia accompanied by epileptic seizures. ${ }^{2,12)}$

Skull lesions can be divided into three types sclerotic, cystic, and pagetoid - on the basis of $x$ ray findings. ${ }^{4}$ Fibrous dysplasia of the cranial vault is usually of the cystic type. Skull $\mathrm{x}$-rays usually show a radiolucent mass with a sclerotic margin, diploic widening, bulging of the outer table, and little or no involvement of the inner table ${ }^{8)} \mathrm{CT}$ more clearly reveals an intradiploic, multilocular lesion separated by bony trabeculae." Bone scintigraphy often reveals abnormal uptake. ${ }^{3,5,7)}$

Although angiographic abnormalities are very rarely seen in cases of fibrous dysplasia of the cranial vault, in our case external carotid angiography disclosed a faint tumor stain, with feeding from the middle meningeal artery. In a few other reported cases, ${ }^{6,10}$ tumor staining, with feeding from branches of the external carotid artery, was identified.

Treatment of monostotic fibrous dysplasia of the cranial vault depends on age and the type of disease. Adults with inactive disease often require no treatment unless they develop mass signs or neurological symptoms. However, in younger patients and those with active disease, it is advisable to resect the lesion and perform cranioplasty in order to arrest the disease, eliminate neurological symptoms, and/ or correct cosmetic deformities and to confirm the diagnosis histologically.

\section{References}

1) Ameli NO, Rahmat $\mathrm{H}$, Abbassioun $\mathrm{K}$ : Monostotic fibrous dysplasia of the cranial bones: Report of fourteen cases. Neurosurg Rev 4: 71-77, 1981

2) Bertoni JM, Kooi KA: Fibrous dysplasia of the skull, with seizures and focal electroencephalographic findings. Dev Med Child Neurol 20: 483-493, 1978

3) Debois V, Van den Bergh R: Benign tumors of the cranial vault: A report of 12 cases. Clin Neurol Neurosurg 81: 1-12, 1979

4) Fries JW: The roentgen features of fibrous dysplasia of the skull and facial bones: A critical analysis of thirty-nine pathologically proved cases. Amer $J$ Roentgen 77: 71-88, 1957

5) Higashi $T$, Iguchi $M$, Shimura A, Kruglik GD: Computed tomography and bone scintigraphy in polyostotic fibrous dysplasia: Report of a case. Oral Surg 50: 580-583, 1980

6) Ito H, Waga S, Sakakura M: Fibrous dysplasia of the skull with increased vascularity in the angiogram. Surg Neurol 23: 408-410, 1985

7) Kieffer SA, Loken MK: Positive "brain" scans in fibrous dysplasia and other lesions of the skull. Amer $J$ Roentgen 106: 731-738, 1969

8) Leeds N, Seaman WMB: Fibrous dysplasia of the skull and its differential diagnosis: $A$ clinical and roentgenographic study of 46 cases. Radiology 78 : 570-582, 1962

9) Lichtenstein L: Polyostotic fibrous dysplasia. Arch Surg (Chicago) 36: 874-898, 1938

10) Lin JP, Goodkins R, Chase NE, Kricheff II: The angiographic features of fibrous dysplasia of the skull. Radiology 92: 1275-1280, 1969

11) Nager GT, Kennedy DW, Kopstein E: Fibrous dysplasia. A review of the disease and its manifestations in the temporal bone. Ann Otol Rhinol Laryngol [Suppl] 92: 1-52, 1982

12) Nishioka $K$, Masuda $Y$, Inokuchi I, Iyoda $K$, Tanaka T: A case of monostotic fibrous dysplasia of the temporal bone associated with epileptic seizure. Acta Med Okayama 36: 453-462, 1982

13) Sassin JF, Rosenberg RN: Neurological complications of fibrous dysplasia of the skull. Arch Neurol (Chicago) 18: 363-369, 1968

Address reprint requests to: S. Otsuka, M.D., Department of Neurosurgery, Kobe City General Hospital, 4-6 Minatojima-naka-machi, Chuo-ku, Kobe 650, Japan.

Neurol Med Chir (Tokyo) 29, March 1989 